\title{
Analysis of Single-Electrode EEG Rhythms Using MATLAB to Elicit Correlation with Cognitive Stress
}

\author{
Chee-Keong Alfred Lim and Wai Chong Chia
}

\begin{abstract}
This paper demonstrates electroencephalogram (EEG) analysis in MATLAB environment with the objective to investigate effectiveness of cognitive stress recognition algorithm using EEG from single-electrode BCI. 25 subjects' EEG were recorded in MATLAB with the use of Stroop color-word test as stress inducer. Questionnaire on subjects' self-perceived stress scale during Stroop test were gathered as classification's target output. The main analysis tool used were MATLAB, coupled with the use of Discrete Cosine Transform (DCT) as dimension reduction technique to reduce data size down to $2 \%$ of the origin. Three pattern classification algorithms' - Artificial Neural Network (ANN), k-Nearest Neighbor (KNN) and Linear Discriminant Analysis (LDA) were trained using the resulted $2 \%$ DCT coefficients. Our study discovered the use of DCT along with KNN offers highest average classification rate of $72 \%$ compared to ANN and LDA.
\end{abstract}

Index Terms-BCI, EEG, MATLAB, stress recognition.

\section{INTRODUCTION}

We generally use the word "stress" to describe when we feel everything seems to have become too much, strain or under pressure [1]. However, there is more to that, the effect of stress can leads to many psychological illnesses, such as depression, sleep disorder and anxiety [2], [3]. Early detection of stress can therefore reduce the risk of mental illness by taking appropriate stress relief therapies. Stress is also an important factor in mental illness, as it can worsen symptoms of mental illness and lead to relapses [4], thus, the decrement of stress can speed up mental illness' recovery rates.

As mental illness rises in United States and United Kingdom [5], [6], mental health care eventually became a demand. People began to seek for financially feasible and effective mental health care approaches [7]. However, traditional counselling requires individual's willingness in participation to be effective, and many who need counselling may not seek for it. This give rise to the study of electroencephalogram (EEG), a bio-signal recorded from scalp that provides information on human cognitive activity through the measure of neurons' electrical impulses [8]. Self-diagnosis is also made possible with consumer BCI that are currently available for the public. With the wide applications of EEG, all that left now is to identify the unique

Manuscript received March 9, 2014; revised May 13, 2014.

Chee-Keong Alfred Lima and Wai Chong Chia are with the Faculty of Science and Technology, Sunway University, Selangor, Malaysia (e-mail: 09065434@imail.sunway.edu.my; waichongc@ sunway.edu.my). patterns of such brainwaves correspond to various mental states. Through EEG monitoring, individual's stress level can be detected and quantified in an efficient manner.

Our study focuses on evaluating to what extent a single-electrode EEG headset - NeuroSky MindWave is able to classify brainwave in terms of subject's stressor level. By reducing the number of electrodes needed, it also means cheaper EEG headset can be used to diagnose various mental disorders. This would then allow the public and financial infeasible individuals to gain access to mental state diagnosis. With its optimistic outcome, access to chronic stress diagnosis would then be much affordable and easy to implement.

\section{LITERATURE SURVEY}

\section{A. Electroencephalography}

The first report on electrical brain activity in humans published in 1929, allowed clinicians and scientists to watch the brain in action in a meaningful way [9]. Electroencephalography also known as EEG is a recording of the electrical activity of the brain from the scalp, which reflects the neuron-electrical activity [10]. EEG is generally described in terms of its frequency band. Vary of amplitude and frequency of the wave represent various brain states [9], which depends on external stimulation and internal mental states [11].

\section{B. Relevant Researches}

The NeuroSky MindWave is a low cost single-electrode EEG headset, and it has been proven effective in detecting user's mental states [12]. The study done by [12] with the aim to evaluate the effectiveness of NeuroSky MindWave's meditation eSense algorithm had applied the Stroop Color-Word Interference test [13] - a well-known psychological test of selective attention, often utilized as a psychological or cognitive stressor. Each subject's meditation level output were recorded during the Stroop test, with incorrect responses time-stamped. The meditation level timeline was then compared with the error timeline through observation and found no correlation between meditation level and erroneous responses. It is then assumed that the meditation reading of the headset does not register the error made by subject instantaneously.

Another reference [14] directed to classify nine-electrodes EEG signals of university students before and after their examination period had used the Perceived Stress Scale 14 questionnaire (PSS-14) to categorize subjects into stressed and stress-free groups. Subjects' EEG were then classified based on questionnaire's outcome into two groups. 


\section{MATLAB}

By utilize modern computer's computation power, and abundant memory; EEG data can be collected, processed and made meaningful through analysis. Reference [15] had demonstrated the capability of MATLAB in processing EEG signal. EEG machine's electrodes are placed on the head of the subjects with wires that transmit all electrical activity to a computer. However, note that EEG is still a type of electrical signals, which means it is susceptible to noise, artifacts, and interference. With the assistance of MATLAB, these issues can be easily tackled by implementing signal-filtering algorithm, such as removing low frequency components in frequency domain of the signals. Moreover, MATLAB's signal processing functions can be utilized to make these signals more readable and easier to be analyzed. Additionally, data classification with the use of various supervised machine learning algorithms in MATLAB environment were discussed in this paper. Which, in return, exemplify the utility of EEG and MATLAB's applicability in computational biology that serve as a powerful numerical computing environment application.

\section{METHODOLOGY}

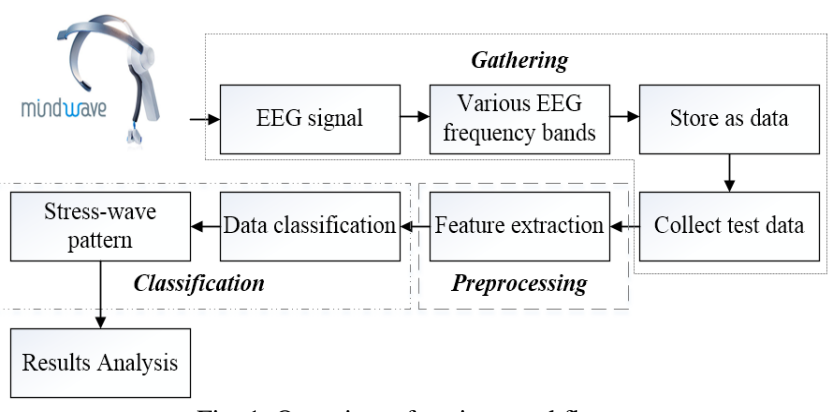

Fig. 1. Overview of project workflow.

Generally, our study can be divided into three main stages as depicted in Fig. 1, and they will be explained in detail in the following subsections.

\section{A. Data Gathering}

$\mathrm{d}=$ red $\quad \mathrm{f}=$ green $\quad \mathrm{j}=$ blue $\quad \mathrm{k}=$ black

\section{green}

Fig. 2. Stroop test implemented.

The NeuroSky's single-electrode MindWave EEG headset is adopted in our study. MATLAB script has been written to retrieve EEG data in real-time through the API provided by NeuroSky. With the objective to identify stress-wave pattern from data collected, test subjects must be induced with a minor level of stress. To achieve that, Stroop Color-Word Interference Test [13] would be used as a cognitive stressor. 30 seconds were allocated for instruction reading, followed by 60 seconds of Stroop test, total 90 seconds of EEG recorded per subject.

The Stroop Color-Word Interference Test is implemented by using Inquisit Lab [16] software. With script written to display color words on screen, and accept user's response through keyboard, with key D, F, J, and K referring to red, green, blue, black color respectively, as shown in Fig. 2.

25 Sunway University students were chosen randomly to attend the Stroop test [13] in an enclosed unoccupied classroom. With their questionnaire's responses on self-perceived stress level rated on a scale from 1 to 10 recorded.

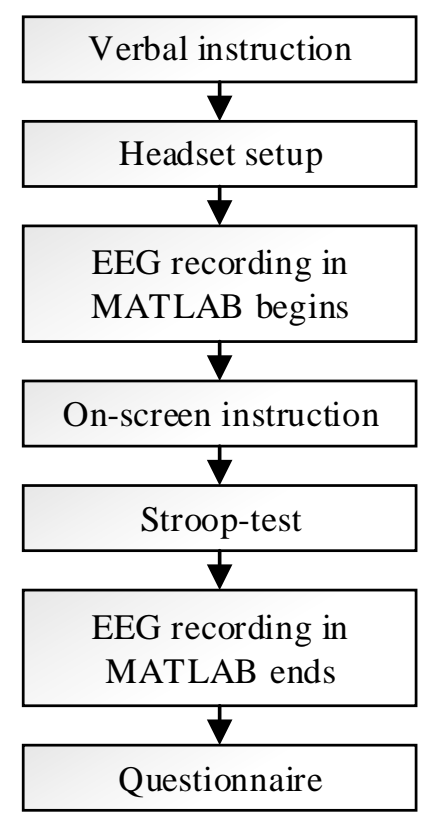

Fig. 3. Data collection procedures.

\section{B. Data Preprocessing}

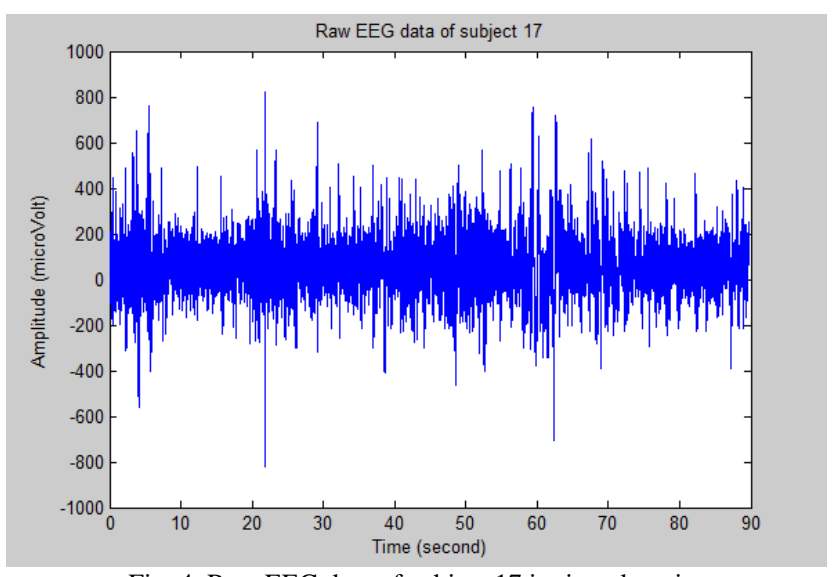

Fig. 4. Raw EEG data of subject 17 in time domain.

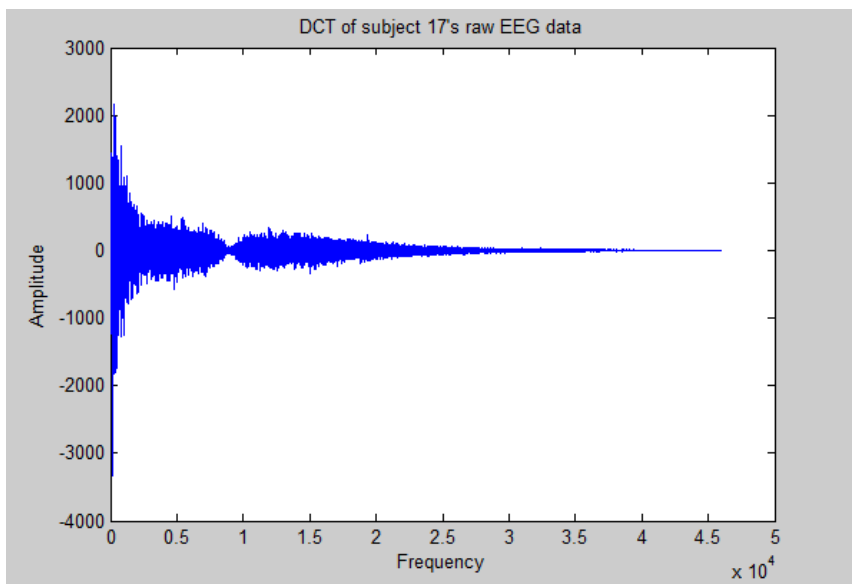

Fig. 5. Subject 17's raw EEG data in frequency domain. 
Discrete Cosine Transform (DCT) is utilized to transform the signal from time-domain to frequency-domain linearly [17]. This allowed us to identify low-frequency components easily, which often contain essential information. While high-frequency components that comprise of noise can be discarded [18], which in-turn provide us noise-filtering feature. Apart from using DCT in noise filtering, its frequency-domain transformation nature also concentrates correlated data's energy in fewer coefficients [19], the resulted signal is much more concentrated, where energy is compressed into first few coefficients and others are relatively small. This provide us essential feature extraction feature as well as noise filtering.

Fig. 4 shows the raw EEG data recorded from subject 17 in time-domain. We then perform DCT on the same data set and the result is shown in Fig. 5. The resulting signal is much more concentrated, where energy is compressed into first few coefficients and others are relatively small. These small coefficients can be omitted from classification [20], which in turn reduce the data size and retain low frequency components.

\section{Data Classification}

Once EEG data were collected from 25 test subjects, these data are then classified by using various type of classifiers in MATLAB. Including Artificial Neural Network (ANN), Linear Discriminant Analysis (LDA) [21], [22], and KNearest Neighbor (KNN), which had been proven to be effective in solving EEG related problems [18], [20], [23], [24]. Therefore, we then evaluate all three classifiers performance using the leave-one-out cross-validation (LOOCV) and k-fold cross-validation.

\section{CLASSIFIERS' IMPLEMENTATIONS}

\section{A. Artificial Neural Network (ANN)}

Artificial Neural Network (ANN) is a type of supervised machine learning algorithm that contains several hidden neuron [23], [25]. Each neuron has its specific weight that may change according to the training data [25]. As the weight of each neuron adapt to the data, the ANN learns the relationship between input and output values, this eventually leads to pattern recognition.

From Fig. 6, we can see that the validation error fluctuates greatly as the number of neuron increases. This phenomenon could be due to the small data set we had, which hindered its precision. Nonetheless, we can still observe its linear trend-line shows a decrement of validation error as number of neuron increased. Validation error were computed after every epoch to avoid over-fitting as neuron increases.

The Neural Network Toolbox provided in MATLAB [26] is utilized to initialize the network. However, due to the memory restriction of MATLAB, we are unable to train an ANN using variables with size of 46080 (90 seconds * 512 sampling rate). Therefore, feature extraction techniques is required to reduce variable size [19]. We used the existing Discrete Cosine Transform (DCT) function in MATLAB [17] to achieve energy compaction on the EEG data collected.

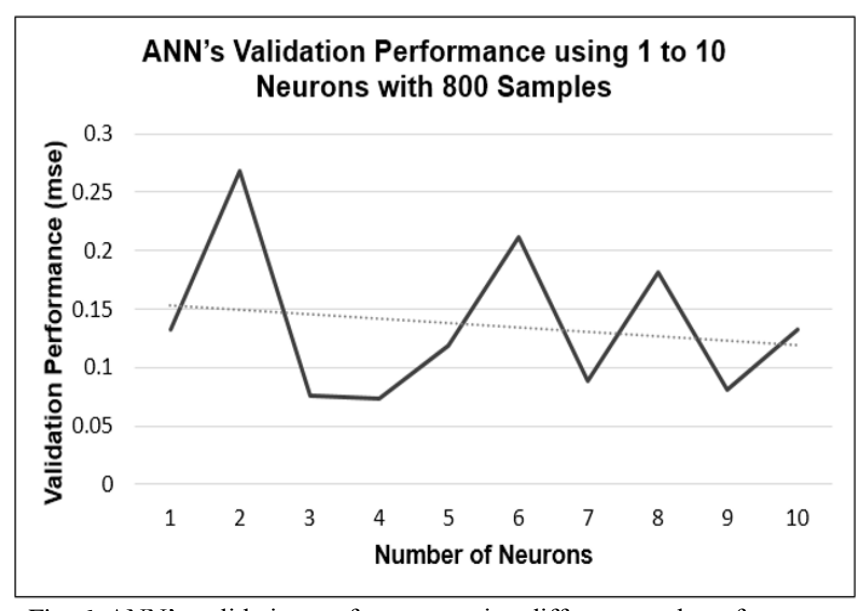

Fig. 6. ANN's validation performance using different number of neurons.

Ref. [18] had demonstrated using only $2 \%$ of the original EEG signal after DCT. Its outcome surpassed the highest accuracy classification algorithm presented in BCI Competition II. Using [18] as a reference, $2 \%$ of 46080 samples would give us 920 samples approximately. As the variable size reduced, we were then able to increase the number of hidden neurons in the ANN to 20, which was demonstrated in [20] to be the best setup for classifying EEG. The ANN's topology used in our study is as shown in Fig. 7.

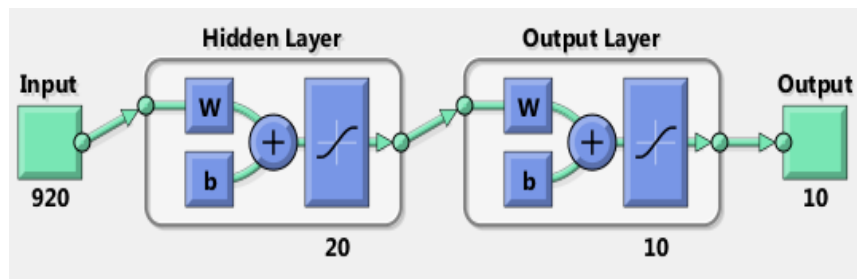

Fig. 7. ANN's topology configuration.

\section{B. Linear Discriminant Analysis (LDA)}

Linear discriminant analysis (LDA) [21] is another widely used machine learning algorithm. The advantage of LDA is its implementation simplicity, where a linear combination of features is used to separate classes of samples [27]. Then again, this is its disadvantage as well, especially when class differences is low. In such case, LDA tend to over-fit the data as it assumes the mean as discriminating factor and not variance [28]. Nevertheless, its implementation is simple, which can be invoked through statistics toolbox in MATLAB environment.

\section{K-nearest Neighbor (KNN)}

$\mathrm{K}$-nearest neighbor (KNN) classifier works in a simple way by comparing both test and training data based on its nearest values [29]. In this case, ' $k$ ' here refers to how many nearest value should be considered before the output class is decided. For instance, if $\mathrm{k}=3$, three nearest points between training and test data will be considered, and its final output belongs to the training data's class with most nearest points [30].

Implementation of $\mathrm{KNN}$ was accomplished using "ClassificationKNN" class in MATLAB's statistic toolbox. Various settings were given to customize the classification behavior of KNN, which include its output decision given that the same amount of closest point to multiple classes 
happened. In our case, the classifier uses random tiebreaker to assign the input data to a tied group randomly with the use of Euclidean distance as distance metric to compute data points' nearest neighbor. The said configuration has proven to provide high accuracy in classifying human stress [29]. Apart from its distance metric and tiebreaker rules, numbers of nearest neighbors to consider during classification is another vital setting. Through cross-validation, k-value of 2 is found to produce lowest average absolute error.

\section{Test Data}

Using MATLAB scripts developed, we were able to read and store EEG data in MATLAB environment from the NeuroSky MindWave headset. All 25 Sunway University students' EEG data were collected for a duration of 90 seconds each. With beginning 30 seconds allocated for on-screen instruction reading, followed by 60 seconds of Stroop test. All subjects' self-perceived stress level responses are summarized in Fig. 9. It is assumed that all subjects' psychological stress were reflected in the EEG data collected, within a comparative features model that correlates to subject's self-perceived stress level.

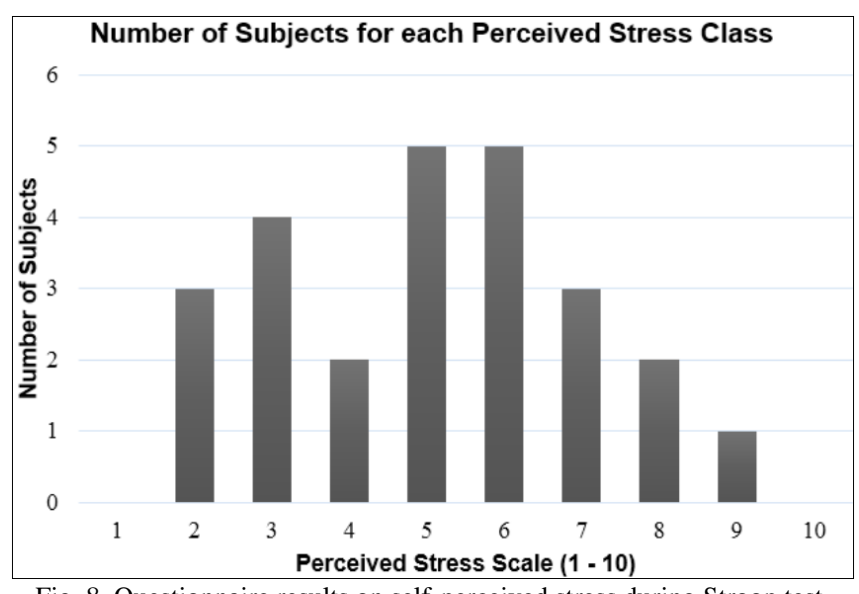

Fig. 8. Questionnaire results on self-perceived stress during Stroop test.

Based on the results obtained in Fig. 8, a mean stress scale of 5 is obtained with most rated perceived stress at level 5 and 6. In addition, none of the subjects had rated themselves under stress scale of 1 and 10. Such outcome is to be expected, as the nature of our experiment requires subjects' attention to operate which contributes at least certain level of cognitive load. Meanwhile, the Stroop test is not stressful to an extent where scale of 10 will be rated.

\section{RESULTS AND DISCUSSIONS}

\section{A. Data Truncation}

A study conducted to evaluate athlete's anxiety score before and after a competition found that human generally experienced higher anxiety before the performance [31]. Similarly, the amount of stress experienced by subjects may vary across the 90 seconds of EEG recording, depending on the subjects' activity at that time. By reducing the scope of EEG for classifier training, it can improve its performance and accuracy. As such, an interview with 5 randomly picked subjects that participated in our EEG data collection were conducted, aiming to understand the time frame during Stroop test that contributed most stress.

The interviews were conducted informally, so that subjects' responses were not influenced by the environment setting and formal procedures. However, the objective of the interview were given to ensure interviewees understand the value of their responses.

TABLE I: INTERVIEW OUTCOME ON STRESS LEVEL VARIATION DURING

\begin{tabular}{|l|c|}
\multicolumn{2}{c|}{ DATA COLLECTION } \\
\hline $\begin{array}{l}\text { Instruction Reading } \\
(30 \text { seconds) }\end{array}$ & Number of Response (5 subjects) \\
\hline $\begin{array}{l}\text { Stroop Test } \\
(60 \text { seconds) }\end{array}$ & 5 \\
\hline
\end{tabular}

An open question of "did you experience most stress during on-screen instruction reading, or the actual Stroop test response period?" Five out of five subjects interviewed had stated that they felt most stress during the instruction reading period. Further clarification on the causes, such as not having enough time to finish the instruction, trying to remember the keys and colors association, and unsure of how Stroop test is conducted using laptop, were provided by interviewees.

All interviewees agreed they generally experienced most stress during the beginning 30 seconds of on-screen instruction reading. To further validate the variation of classification performance using EEG data at different time frames, we began hypothesis testing through ANN classification by dividing the data into two groups: first 30 seconds, and trailing 60 seconds.

TABLE II: CLASSIFIERS` OUTCOME USING STRESS SCALE OF 1 TO 10

\begin{tabular}{|l|c|c|}
\hline & $\begin{array}{c}\text { Beginning 30 } \\
\text { seconds }\end{array}$ & $\begin{array}{c}\text { Trailing 60 } \\
\text { seconds }\end{array}$ \\
\hline Mean absolute error (MAE) & $\mathbf{2 . 2}$ & 2.68 \\
\hline
\end{tabular}

LOOCV [32] were then conducted 25 times for each subject's EEG. Result shows that using the beginning 30 seconds of the EEG data in training ANN would produce higher classification accuracy when compared to the trailing 60 seconds. This affirmed the interview's outcome on subjects generally experienced more stress during the on-screen instruction reading, which is the beginning 30 seconds prior to begin of the Stroop test. Hence, the beginning 30 seconds is truncated for classification.

\section{B. Data Classification}

The beginning 30 seconds of the 25 subjects' EEG data were then preprocessed using DCT with the aim to extract its features. Based on subjects' perceived stress scale, classification is then performed using ANN, LDA, and KNN classifiers that were implemented in MATLAB by using the existing toolbox and libraries.

\begin{tabular}{|l|c|c|c|}
\hline TABLE III: CLASSIFIERS' OUTCOME USING STRESS SCALE OF 1 TO 10 \\
\hline $\begin{array}{l}\text { Misclassification Rate } \\
(\%)\end{array}$ & $\mathbf{6 N N}$ & LDA & KNN \\
\hline $\begin{array}{l}\text { Mean absolute error } \\
\text { (MAE) }\end{array}$ & 2.2 & 84.0 & 96.0 \\
\hline
\end{tabular}


Table III shows the summarized outcome of each classifiers. Note that the misclassification rates (MR) were computed using confusion matrix, and mean absolute error (MAE) were acquired through 25 epochs of LOOCV. From the results, we observed that ANN provides lowest MR, while KNN offers smallest MAE. This resulted in a contrast relationship between MR and MAE, whereby as the classifier's MR increases, its MAE decrease. To illustrates the phenomena better, we have scaled the MAE $(* 10)$ in Fig. 9 to highlights its differences across various classifiers.

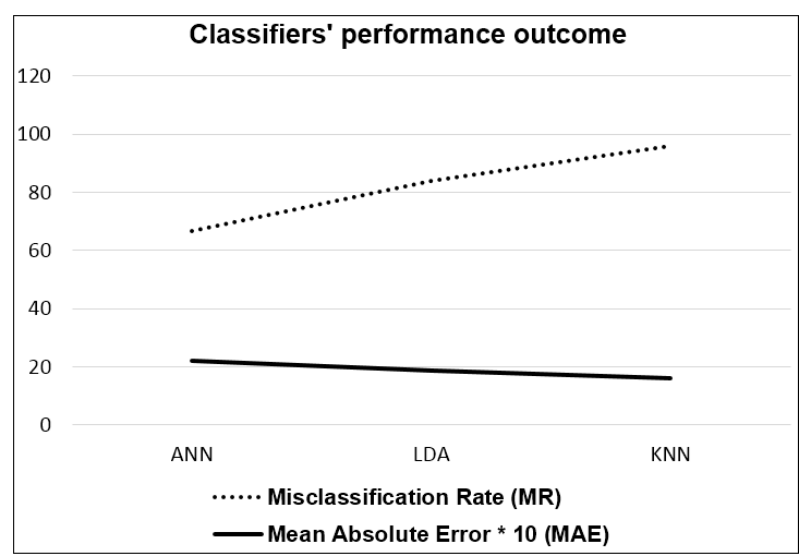

Fig. 9. Performance outcome for each classifiers in terms of its MR and MAE.

MAE is calculated by finding the difference between classifier's actual and desired output [33]. Thus, classifier that generates relative higher MAE implies its output deviate greater from the desired output. On the other hand, MR provides us the evidence on classifier's incorrect predictionnumbers of sample that were classified incorrectly.

Consequently, a classifier can produce high MR with low MAE or vice-versa, with no illogicality. For instance, KNN is able to achieve lowest MAE with highest MR. As both performance metric measure a classifier's performance in a different perspective. In general, MR measures how often a classifier goes wrong while MAE measures how wrong a classifier goes in average.

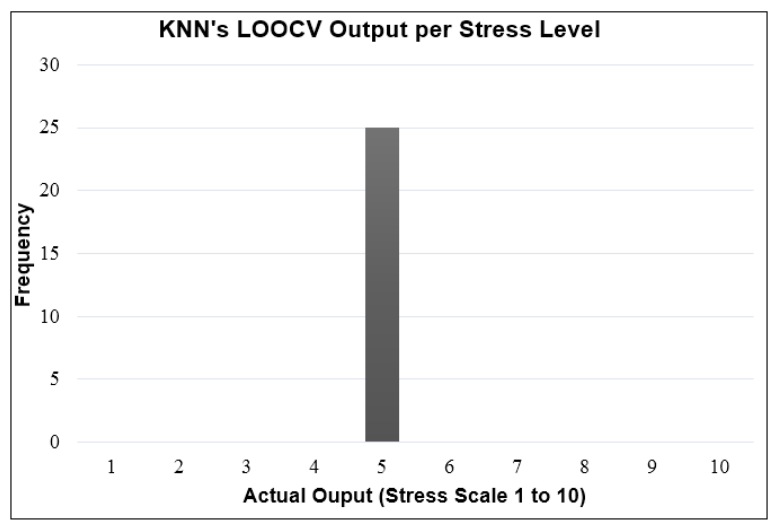

Fig. 10. KNN's actual output for all 25 subjects' self-perceived stress.

It is important to note that the perceived stress scale measured were by best effort. In other words, there is no guarantee that a subject rated stress scale of 5 actually experienced the same amount of stress with other subjects that rated the same. Therefore, we are interested in minimizing the MAE rather than MR. Then again, it is unrealistic to measure a classifier's performance solely based on its computed MAE results [34]. This is owing to classifier can achieved minimal MAE by sustaining its output close to target class' mean value as showed in Fig. 10.

According to Fig. 8, the mean perceived scale class were 5. Using output class of 5, we were able to achieve 1.6 MAE as shown in Table III. By comparing the MR and MAE of all three classifiers, we have discovered the disadvantage of using MAE as performance metric in our study [33] due to the distribution of data. MAE is minimized when classifier output were sustained at mean target class value, which does not reflects the true error rate of a classifier. Despite the outcome MAE is fairly low, but in reality, the classifier was merely minimizing the error by classifying all input data to its average target class. This proven that MAE is not a reliable performance metric for our study.

While MR is not a suitable performance metric either, due to stress scale is measured by best effort as a subjective emotional experience, which is difficult for classifier to identify the correlation between data features and its target class. We then assess the cause of high MAE by categorizing stress level into two classes - ' 1 ' for non-stressed, and ' 2 ' for stressed (see Table IV).

TABLE IV: STRESS LEVEL SCALING REFERENCE TABLE

\begin{tabular}{|c|c|c|}
\hline $\begin{array}{c}\text { Actual } \\
\text { Value }\end{array}$ & $\begin{array}{c}\text { Scaled } \\
\text { Value }\end{array}$ & Linguistic Variable (Stress level) \\
\cline { 1 - 1 } 1 & & \\
\cline { 1 - 1 } 2 & 1 & Non-stressed \\
\cline { 1 - 1 } 3 & & \\
\cline { 1 - 1 } 4 & & \\
\cline { 1 - 1 } 5 & & \\
\cline { 1 - 1 } 6 & & \\
\cline { 1 - 1 } 7 & & \\
\cline { 1 - 2 } 9 & & \\
\cline { 1 - 2 } & & \\
\hline
\end{tabular}

Classifiers were then trained using the scaled target class, and cross-validated using leave-one-out cross-validation (LOOCV) [35]. The corresponding results of all test sets are presented in Table V.

TABLE V: ClASSIFIER'S PERFORMANCE WITH SCALED STRESS LEVEL

\begin{tabular}{|l|c|c|c|}
\hline & ANN & LDA & KNN \\
\hline $\begin{array}{l}\text { Misclassification Rate } \\
(\boldsymbol{\%})\end{array}$ & 56 & 40 & $\mathbf{2 8}$ \\
\hline LOOCV & 0.56 & 0.40 & $\mathbf{0 . 2 8}$ \\
\hline
\end{tabular}

$\mathrm{KNN}$ is able to achieve $72 \%$ accuracy, which performs much better than ANN (44\%) and LDA (60\%). With the target class scaled down to only two output ( $1=$ non-stressed, 2 = stressed), KNN's performance improved significantly compared to previous result (see Table III), while ANN and LDA's performance deteriorated. However, such phenomena is anomalous. As the target class is scaled down, classifier's performance should be higher due to the exclusion of having a precise stress level classification. This experiment had demonstrated that both ANN and LDA are less reliable 
compared to KNN in classifying EEG based human stress. To further validate the accuracy of $\mathrm{KNN}$, a 5-fold cross-validation is performed.

Using k-fold validation, the $\mathrm{k}$ value determines the number of partition that samples should be divided. Depending on the number of partitions, the same number of cross-validate iteration would then be performed, each with a unique partition as test set. In other words, if $k=25$, then $\mathrm{k}$-fold validation would became LOOCV in our case, as there are total 25 samples.

We adopted $80: 20$ ratio, where by in every training instance, 20 samples (80\%) were used as test set, while the other 5 samples $(20 \%)$ as train set. To achieve the said partitioning ratio, $\mathrm{k}$ value is set to $5(25 / 5=5)$. Using scaled target class and 512 DCT coefficients of subjects' beginning 30 seconds EEG, a k-fold cross-validation [36] is then performed on KNN. The estimated average outcome is as follows (see Table VI):

TABLE VI: KNN's 5-FOLD CROSS-VALIDATION CONFUSION MATRIX

\begin{tabular}{|c|c|c|c|}
\cline { 3 - 4 } \multicolumn{2}{c|}{} & \multicolumn{2}{c|}{ Target Class } \\
\cline { 3 - 4 } Output & $\mathbf{1}$ & 14 & $\mathbf{2}$ \\
\cline { 3 - 4 } Class & $\mathbf{2}$ & 0 & 4 \\
\hline
\end{tabular}

From the confusion matrix (see Table VI), we can compute its accuracy using $[(\mathrm{TP}+\mathrm{TN}) /(\mathrm{TN}+\mathrm{FP}+\mathrm{FN}+\mathrm{TP})]$, where TP referred as True Positive and FN as False Negative [32]. This resulted in $72 \%$ accuracy, which is the same as our previous LOOCV's outcome (see Table V). Thus, affirmed the capability of KNN in classifying human stress.

\section{CONCLUSION AND FUTURE WORK}

This paper demonstrated the procedures of EEG signal processing in MATLAB environment. With proper scripting, data can be retrieved from BCI and processed numerically by utilize modern computing power. Although that hardware specification may pose several restriction on the data size, nonetheless it can be resolved by fine-tuning the implementation approach and make use of several existing signal-processing algorithms. We demonstrated the use of Discrete Cosine Transform (DCT) as feature extraction technique coupled with KNN classifier, and were able to obtain $72 \%$ accuracy in classifying EEG that correlated to human stress. Despite the EEG data were collected using a single electrode BCI - NeuroSky MindWave, yet the classification outcome were not far behind than multi electrode BCI [15], [18], [20], [25], [37], [38]. With proper choice of classifier and feature extraction components, it is possible to employ a single electro BCI in EEG research and recognizes human stress. Not only has it reduced the cost of implementation, but also provide a choice of compact EEG headset to be used in public.

Future work aiming to increase the classification accuracy of human stress using EEG and NeuroSky MindWave can too be extended from our study. With the use of MATLAB scripting and NeuroSky developer toolkit, investigation on a more detailed EEG frequency band, such as the Alpha, Beta and Gamma wave; can potentially increase classifier's accuracy owing to the greater EEG features provided. Furthermore, Discrete Wavelet Transform (DWT) that make use of vary window size depending on the fluctuation of signal within a time period can be a useful alternative to DCT [29]. The nature of DWT tends to retains more information than DCT [39], which can prevent the loss of stress wave features during feature extraction phase. Thus, able to provide a more distinct data attributes for classifiers to adapt and recognize. Additionally, the measure of stress level using self-perceived stress scale is by best effort, the actual stress amount experienced by subjects may differ. Data accuracy therefore can be improved via alternate stress measurement, such as biochemical response - salivary cortisol hormone, blood pressure, or heart rate [40].

\section{ACKNOWLEDGMENT}

The first author, Chee-Keong Alfred Lim, would like to acknowledge the good support given by staffs in Faculty of Science and Technology, Sunway University, Malaysia.

\section{REFERENCES}

[1] C. Nordqvist, What Is Stress? How to Deal with Stress, MediLexicon International Ltd, April 11, 2009.

[2] The National Institute of Mental Health. NIMH. The numbers count: mental disorders in America. The Natinal Institute of Mental Health. [Online]. Available: http://www.nimh.nih.gov/health/publications/the-numbers-count-ment al-disorders-in-america/index.shtml

[3] M. Smith, R. Segal, and J. Segal. (April 2013). Stress symptoms, signs $\&$ causes: effects of stress overload. [Online]. Available: http://www.helpguide.org/mental/stress_signs.htm

[4] U. S. Department of Health and Human Services, "Illness managemen and recovery, an evidence-based practices," Substance Abuse and Mental Health Services Administration, 2010.

[5] T. Latham, "Mentail Illness on the rises in the U.S.," Psychology Today, Sussex Publishers, LLC, May 18, 2011.

[6] N. O. Brien. The remarkable rise of mental illness in Britain Telegraph Blogs. Telegraph Media Group Limited. (October 30, 2012). [Online]. Available: http://blogs.telegraph.co.uk/news/neilobrien1/100186974/the-remarka ble-rise-of-mental-illness-in-britain/

[7] J. R. Evans, Handbook of Neurofeedback: Dynamics and Clinical Applications, Haworth Press, 2007.

[8] T. H. Budznski, H. K. Budzynski, J. R. Evans, and A. Abarbanel, Introduction to Quantitative EEG and Neurofeedback: Advanced Theory and Applications, Academic Press, 2009.

[9] D. A. Kaiser. What is quantitative EEG. [Online]. Available: http://www.skiltopo.com/skil3/what-is-qeeg-by-kaiser.pdf

[10] McGill University. EEG. McGill University. [Online]. Available: http://www.medicine.mcgill.ca/physio/vlab/biomed_signals/eeg_n.ht $\mathrm{m}$

[11] NeuroSky Inc.. Brain Wave Signal (EEG) of NeuroSky, Inc. $\begin{array}{lll}\text { (December 2009). [Online]. Available: } & \text { 15, }\end{array}$ http://frontiernerds.com/files/neurosky-vs-medical-eeg.pdf

[12] C. Katie, S. Aidan, P. Ian, and M. Dave, "Evaluating a brain-computer interface to categorise human emotional response," in Proc. 10th IEEE International Conference on Advanced Learning Technologies, 2010, pp. 276-278.

[13] J. R. Stroop, "Studies of interference in serial verbal reactions," Journal of Experimental Psychology, vol. 28, pp. 643-662, 1935.

[14] R. Khosrowabadi, C. Quek, K. K. Ang, S. W. Tung, and M. Heijnen, "A Brain-Computer Interface for classifying EEG correlates of chronic mental stress," in Proc. of International Joint Conference on Neural Networks, vol. 11, 2011, pp. 757-762.

[15] S. Gurumurthy, V. S. Mahit, and R. Ghosh, "Analysis and simulation of brain signal data by EEG signal processing technique using MATLAB," International Journal of Engineering and Technology, vol. 5, no. 3, pp. 2771-2776, 2013.

[16] Millisecond Software. Inquisit by Millisecond Software. [Online]. Available: http://www.millisecond.com/ 
[17] The MathWorks, Inc.. (2013). Discrete cosine transform (DCT). [Online]. http://www.mathworks.com/help/signal/ref/dct.html

[18] D. Birvinskas, V. Jusas, I. Martisius, and R. Damasevicius, "Data compression of EEG signals for artificial neural network classification," Information Technology and Control, vol. 42, no. 3, pp. 238-241, 2013.

[19] C. Aparna, J. V. R. Murthy, R. Babu, and S. Rao, "Energy computation using DCT for brain computer interface motor imagery classification," International Journal of Computer Science \& Engineering Technology, vol. 1, no. 1, pp. 17-20.

[20] D. Birvinskas, V. Jusas, I. Martisius, and R. Damasevicius, "EEG dataset reduction and feature extraction using discrete cosine transform," in Proc. 2012 UKSim - AMSS 6th European Modelling Symposium, 2012, pp. 199-204.

[21] The MathWorks Inc. The MathWorks - Examples - Classification. [Online]. Available: http://www.mathworks.com/products/demos/statistics/classdemo.html

[22] O. Moran. (September 15, 2011). Brainwave feature extraction, classification \& prediction. [Online]. Available: http://www.slideshare.net/oliviamoran/feature-extraction-classificatio n-prediction

[23] R. Eberhart and Y. Shi, "Neural network concepts and paradigm," Computational Intelligence: Concepts to Implementation, Burlington, MA, Elsevier, pp. 190-192, 2007.

[24] V. V. Shete, S. Sonar, A. Charantimath, and S. Elgendelwar, "Detection of K-complex in sleep EEG signal with matched filter and neural network," International Journal of Engineering Research \& Technology (IJERT), vol. 1, no. 3, pp. 1-4, 2012.

[25] S. Marsland, "The Multi-Layer Perceptron," Machine Learning: An Algorithm Perspective, London, Chapman \& Hall/CRC, pp. 47-91, 2009.

[26] The MathWorks, Inc. Neural Network Toolbox - MATLAB. [Online]. Available: http://www.mathworks.com/products/neural-network/

[27] J. Ye, R. Janardan, and Q. Li, "Two-Dimensional linear discriminant analysis," Neural Networks for Signal Processing, vol. 9, pp. 41-48, 1999.

[28] Saarland University. Linear Discriminant Analysis (LDA). [Online]. Available: http://www.lsv.uni-saarland.de/dsp_ss05_chap11.pdf

[29] N. Sulaiman, M. N. Taib, S. Lias, Z. H. Murat, S. A. M. Aris, N. Hayatee, and A. Hamid, "EEG-based stress features using spectral centroids technique and k-Nearest neighbor classifier," in Proc. 2011 UKSim 13th International Conference on Modelling and Simulation, pp. 69-74, 2011.

[30] The MathWorks, Inc. Classify data using nearestneighbor method MATLAB knnclassify. [Online]. Available: http://www.mathworks.com/help/bioinfo/ref/knnclassify.html

[31] M. M. Ciucurel, "The relation between anxiety, reaction time and performance before and after sport competitions," Procedia - Social and Behavioral Sciences, vol. 33, pp. 885-889, 2012.
[32] R. Eberhart and Y. H. Shi, "Performance Metrics," Computational Intelligence: Concepts to Implementations, Burlington, Morgan Kaufmann, pp. 389-420, 2007.

[33] C. J. Willmott and K. Matsuura,"Advantages of the mean absolute error (MAE) over the root mean square error (RMSE) in assessing average model performance," Climate Research, vol. 30, pp. 79-82, 2005.

[34] S. M. McNee, J. Riedl, and J. A. Konstan, "Being accurate is not enough: How accuracy metrics have hurt recommender systems," Extended Abstracts on Human Factors in Computing Systems, pp. 1097-1101, 2006.

[35] G. C. Cawley and N. L. C. Talbot, "Efficient leave-one-out cross-validation of kernel fisher discriminant classifiers," Pattern Recognition, vol. 36, no. 11, pp. 2585-2592, 2003.

[36] The MathWorks, Inc. Generate cross-validation indices - MATLAB crossvalind. [Online]. Available: http://www.mathworks.com/help/bioinfo/ref/crossvalind.html

[37] R. J. Barry, A. R. Clarke, and S. J. Johnstone, "A review of electrophysiology in attention-deficit/hyperactivity disorder: I. Qualitative and quantitative electroencephalography," Clinical Neurophysiology, vol. 114, no. 2, pp. 171-183, 2003.

[38] E. Gallego-Jutgla, M. Elgendi, F. Vialatte, J. Sole-Casals, A. Cichocki, C. Latchourmane, J. Jeong, and J. Dauwels, "Diagnosis of Alzheimer's disease from EEG by means of synchrony measures in optimized frequency bands," in Proc. 2012 Annual International Conference of the IEEE on Engineering in Medicine and Biology Society (EMBC), 2012, pp. 4266-4270.

[39] B. Tewari, S. Dubey, and M. Nizamuddin, "Performance analysis of image compression using wavelets," Industrial Electronics, IEEE Transactions on Industrial Electronics, vol. 48, no. 3, pp. 682-695, 2001.

[40] S. H. Seo and J. T. Lee, "Stress and EEG," Convergence and Hybrid Information Technologies, pp. 413-426, 2010.

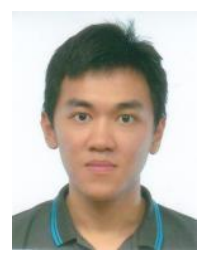

Chee-Keong Alfred Lim is from Petaling Jaya, Selangor, Malaysia. He completed a dual award undergraduate degree of "BSc (Hons) computer science" in 2014 from Sunway University, Malaysia and Lancaster University, UK. His research interests include electroencephalography (EEG), and cognitive neuroscience.

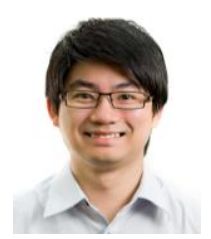

Wai Chong Chia received his MSc in electrical and electronic engineering in 2008 from the University of Nottingham, Malaysia and his Ph.D in 2013 from the same University. He is currently a senior lecturer in the Dept. of Computer Science and Networked Systems in the Faculty of Science and Technology at Sunway University, Malaysia. His research interests include image and video processing, wireless sensor networks, and embedded systems 